\title{
Universal quantum oscillations in the underdoped cuprate superconductors
}

\author{
Neven Barišić ${ }^{1,2,3 \star}$, Sven Badoux ${ }^{4}$, Mun K. Chan ${ }^{2}$, Chelsey Dorow ${ }^{2}$, Wojciech Tabis ${ }^{2}$, \\ Baptiste Vignolle ${ }^{4}$, Guichuan $\mathrm{Yu}^{2}$, Jérôme Béard ${ }^{4}$, Xudong Zhao ${ }^{2,5}$, Cyril Proust ${ }^{4 \star}$ \\ and Martin Greven ${ }^{2 \star}$
}

\begin{abstract}
The metallic state of the underdoped high- $T_{c}$ cuprates has remained an enigma: how may seemingly disconnected Fermisurface segments, observed in zero magnetic field as a result of the opening of a partial gap (the pseudogap), possess conventional quasiparticle properties ${ }^{1-3}$ ? How do the small Fermi-surface pockets evidenced by the observation of quantum oscillations emerge as superconductivity is suppressed in high magnetic fields ${ }^{4-11}$ ? Such quantum oscillations, discovered in underdoped $\mathrm{YBa}_{2} \mathrm{Cu}_{3} \mathrm{O}_{6.5}\left(\mathrm{Y} 123\right.$; ref. 12) and $\mathrm{YBa}_{2} \mathrm{Cu}_{4} \mathrm{O}_{8}$ (Y124; ref. 13), signify the existence of a conventional Fermi surface $^{3,14,15}$. However, owing to the complexity of the crystal structures of $\mathrm{Y} 123$ and $\mathrm{Y} 124\left(\mathrm{CuO}_{2}\right.$ double layers, $\mathrm{CuO}$ chains, low structural symmetry), it has remained unclear whether the quantum oscillations are specific to this particular family of cuprates $^{5,16-18}$. Numerous theoretical proposals have been put forward to explain the source of quantum oscillations, including materials-specific scenarios involving $\mathrm{CuO}$ chains and scenarios involving the quintessential $\mathrm{CuO}_{2}$ planes $^{6,11,18}$. Here we report the observation of quantum oscillations in underdoped $\mathrm{HgBa}_{2} \mathrm{CuO}_{4+\delta}(\mathrm{Hg} 1201)$, a model cuprate superconductor with individual $\mathrm{CuO}_{2}$ layers, high tetragonal symmetry and no $\mathrm{CuO}$ chains. This observation proves that quantum oscillations are a universal property of the underdoped $\mathrm{CuO}_{2}$ planes, and it opens the door to quantitative future studies of the metallic state and of the Fermi-surface reconstruction phenomenon in this structurally simplest cuprate.
\end{abstract}

There exists a broad consensus that the cuprates approach conventional metallic behaviour at very high hole-dopant concentrations ${ }^{19-21}(p)$. For example, $\mathrm{Tl}_{2} \mathrm{Ba}_{2} \mathrm{CuO}_{6+\delta}$ at $p \approx 0.30$ is characterized by a large hole-like Fermi surface that corresponds to approximately $65 \%$ of the first Brillouin zone (containing $1+p$ carriers per $\mathrm{CuO}_{2}$ plane), in good agreement with band-structure calculations ${ }^{15,20,21}$. This is contrasted by the as yet unexplained hightemperature metallic state at intermediate hole concentrations, and by the strong insulating behaviour in the absence of doped carriers. The observation for Y123 (ref. 12) and Y124 (ref. 13) of quantum oscillations not far from the undoped insulating state (for $p \approx 0.1$ ) is surprising, as quantum oscillations are a prominent characteristic of simple conventional metals, and it has triggered much theoretical activity ${ }^{6-11,14,16-18}$. Moreover, the observed oscillation period implies a very small pocket (covering only $\sim 2 \%$ of the zone), in stark contrast to the situation at high hole-dopant concentrations. Although this result can been taken as evidence for a marked change of the Fermi surface associated with the quintessential $\mathrm{CuO}_{2}$ planes, it may also be attributed to the existence of a non-universal Fermi-surface piece related to the $\mathrm{CuO}$ chains in Y123 and Y124 (refs 5,16-18).

In the latter scenario, hybridization between $\mathrm{CuO}$ chain and $\mathrm{BaO}$ plane states gives rise to a small hole pocket ${ }^{16-18}$. This scenario seems to be supported by the observation of a small residual electronic term of the specific heat, and by the persistence of the $\sqrt{ } H$ signature of a $d$-wave superconducting gap to high fields, even on the appearance of quantum oscillations, indicating the coexistence of two very different types of Fermi surface ${ }^{5}$. Consequently, the superconducting properties may be attributed to the large hole Fermi surface due to the $\mathrm{CuO}_{2}$ planes, whereas the quantum oscillations may be associated with pockets present already at low fields. Additional effects due to an electronic instability of the $\mathrm{CuO}$ chains may occur, akin to well-known examples in organic metals where quasi-one-dimensional bands can support spin-density wave order induced by a magnetic field, resulting in quantum oscillations related to the reconstructed closed Fermi surface ${ }^{22}$.

The other class of scenarios invokes symmetry breaking within the $\mathrm{CuO}_{2}$ planes that leads to Fermi-surface reconstruction at low temperature. Indeed, high-field nuclear magnetic resonance measurements revealed static charge-density modulations within the $\mathrm{CuO}_{2}$ planes of underdoped $\mathrm{Y} 123$ (ref. 23). Subsequent X-ray studies of Y123 found charge-density-wave (CDW) correlations ${ }^{24,25}$, with comparable correlation length along and perpendicular to the $\mathrm{CuO}$ chains $^{25}$, consistent with the notion that the quantum oscillations are a property of the $\mathrm{CuO}_{2}$ planes. However, the orthorhombic structure and the chain layers impose a special direction in Y123 and Y124, which might promote and stabilize the charge modulation. Consequently, it has remained a pivotal open question whether the Fermi-surface reconstruction has anything to do with aspects of the unidirectional structures, or whether it is a universal property of the cuprates.

Here we settle this issue through a transverse magnetoresistivity study of $\operatorname{Hg} 1201$ at $p \approx 0.09$ in pulsed magnetic fields of up to $80 \mathrm{~T}$. This doping level is close to that for which quantum oscillations were initially observed in Y123. Figure 1 shows the schematic crystal structures of both compounds. Only samples with sufficiently low scattering rate can show the passage of the Landau levels across the Fermi level. A major obstacle to overcome was the synthesis and electrical contacting of ultrapure single crystals with negligible residual resistivity ${ }^{1}$. This was

\footnotetext{
${ }^{1}$ Service de Physique de l'Etat Condensé, CEA-DSM-IRAMIS, F 91198 Gif-sur-Yvette, France, ${ }^{2}$ School of Physics and Astronomy, University of Minnesota, Minneapolis, Minnesota 55455, USA, ${ }^{3}$ Institute of Physics, HR-10000 Zagreb, Croatia, ${ }^{4}$ Laboratoire National des Champs Magnétiques Intenses, UPR 3228, (CNRS-INSA-UJF-UPS), Toulouse 31400, France, ${ }^{5}$ State Key Lab of Inorganic Synthesis and Preparative Chemistry, College of Chemistry, Jilin University, Changchun 130012, China. ^e-mail:nbarisic@ifs.hr; cyril.proust@lncmi.cnrs.fr; greven@physics.umn.edu
} 

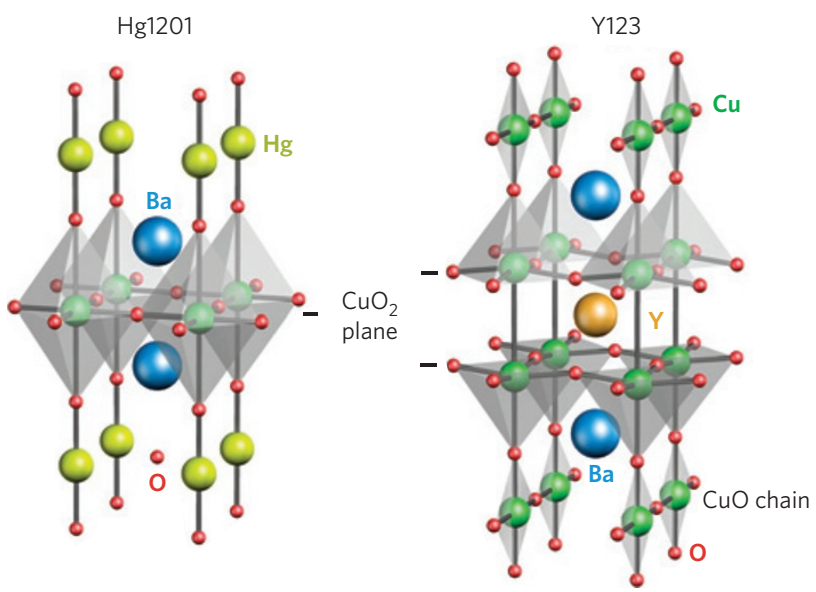

Figure 1 | Crystal structures of Hg1201 and Y123. The two compounds considerably differ in their structural symmetry (tetragonal versus orthorhombic), absence versus presence of $\mathrm{Cu}-\mathrm{O}$ chains, number of $\mathrm{CuO}_{2}$ planes per primitive cell (one versus two), $\mathrm{CuO}_{6}$ octahedra versus $\mathrm{CuO}_{5}$ pyramids, $c$-axis dimensions ( $c \approx 9.53$ versus $11.65 \AA$ ), the most prevalent disorder types, and so on ${ }^{30}$. In $\mathrm{Hg} 1201$, the hole concentration in the $\mathrm{CuO}_{2}$ planes is altered by varying the density of interstitial oxygen atoms in the $\mathrm{Hg}$ layer (indicated schematically), whereas in Y123 it is controlled by the density of oxygen atoms in the $\mathrm{CuO}$ chains (in underdoped $\mathrm{Y} 123$, a significant fraction of chain $\mathrm{O}$ sites is vacant; not shown).

achieved according to previously reported protocols ${ }^{26,27}$ - see also Supplementary Information.

Figure 2 shows isothermal magnetoresistance curves for four different temperatures above the irreversibility field $\left(H_{\text {irr }}=20-30 \mathrm{~T}\right)$. The applied current was parallel to the $\mathrm{CuO}_{2}$ planes, whereas the magnetic field was perpendicular to the planes. The oscillatory behaviour, which is our main result, is already evident from the raw data at fields above $60 \mathrm{~T}$. To emphasize the quantum oscillations without subtraction of the non-oscillatory component, we also plot the derivative of the data (Fig. 2, inset). We note that the oscillations at all four temperatures are in phase with each other. Quantum oscillations with the same frequency were observed in another sample with a similar transition temperature $\left(T_{\mathrm{c}}\right)$, but with lesser precision (see Supplementary Information).

In Fig. 3a, a smooth non-oscillatory contribution is removed and the result is plotted versus $1 / B$. The Fourier transforms in the limited field range [ $62 \mathrm{~T}, 81 \mathrm{~T}]$ exhibit a single peak at $F=840 \pm 30 \mathrm{~T}$ (Fig. 3b). According to the Onsager relation, $F=A_{k} \Phi_{0} / 2 \pi^{2}$, where $\Phi_{0}$ is the magnetic flux quantum and $A_{k}$ is the cross-sectional area of the Fermi surface perpendicular to the applied field, which corresponds to about 3\% of the Brillouin zone. Assuming that the Fermi surface is strictly two-dimensional, the Luttinger theorem yields $n_{2 \mathrm{D}}=2 A_{k} /(2 \pi)^{2}=F / \Phi_{0}=0.061 \pm 0.002$ carriers per pocket. In a single-band model, the Hall coefficient $R_{\mathrm{H}}=1 / n_{3 \mathrm{D}} e\left(n_{3 \mathrm{D}}=n_{2 \mathrm{D}} / c\right.$, where $c$ is the lattice parameter perpendicular to the $\mathrm{CuO}_{2}$ planes) can be evaluated to be $\left|R_{\mathrm{H}}\right|=14.7 \pm 0.6 \mathrm{~mm}^{3} \mathrm{C}^{-1}$, in very good agreement with the value $\left|R_{\mathrm{H}}\right|=15 \pm 5 \mathrm{~mm}^{3} \mathrm{C}^{-1}$ obtained at low temperatures and high fields for a Hg1201 sample with a similar doping level $\left(T_{\mathrm{c}}=65 \mathrm{~K}, p \approx 0.075\right.$; ref. 28). By following the temperature dependence of the oscillation amplitude (Fig. 3b, inset), the quasiparticle effective mass $m^{*}=2.45 \pm 0.15 m_{\mathrm{e}}$ is extracted (where $m_{\mathrm{e}}$ is the free-electron mass). By performing a Lifshitz-Kosevich fit (solid lines in Fig. 3a), the Dingle temperature can be evaluated to be $T_{\mathrm{D}}=(18 \pm 4) \mathrm{K}$, which corresponds to a mean free path of $\ell \approx 5 \mathrm{~nm}$.

The quantum oscillations in Y123 and Hg1201 appear approximately in the same doping, temperature and magnetic field range of the phase diagram, with pockets of very similar effective mass

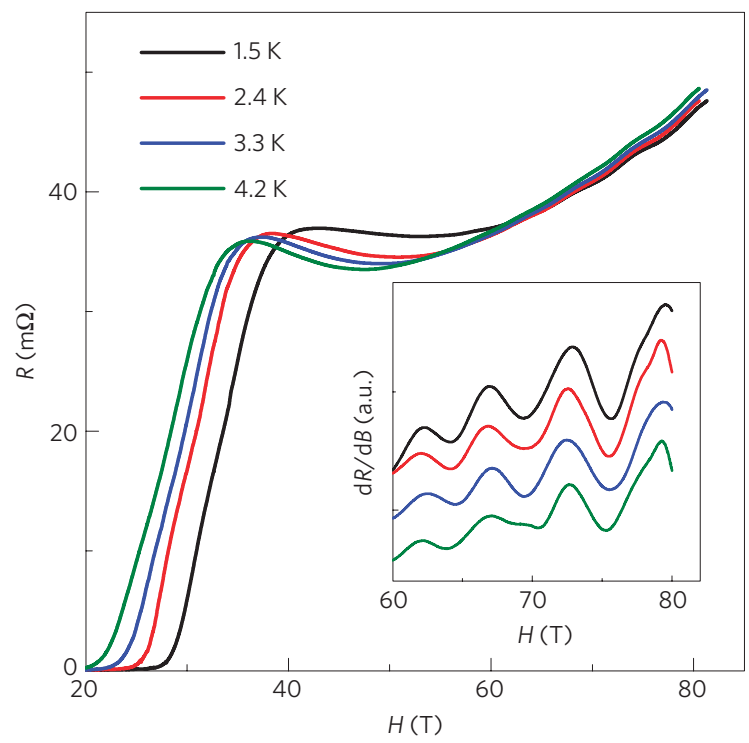

Figure 2 | Quantum oscillations in Hg1201. Planar transverse magnetoresistance data for a $\mathrm{Hg} 1201$ sample (zero-field $T_{\mathrm{C}}=72 \mathrm{~K}$, $p \approx 0.09$ ) in pulsed magnetic fields (up to $80 \mathrm{~T}$ ) at selected temperatures $(1.5,2.4,3.3$ and $4.2 \mathrm{~K})$. Quantum oscillations are clearly observed from the raw data above $60 \mathrm{~T}$. Inset: the derivative of the resistivity highlights the oscillations.

and comparable cyclotron frequency, as summarized in Table 1. Moreover, the temperature dependence and absolute value of the Hall and Seebeck coefficients are nearly the same for $p \approx 0.09$. Remarkably, on cooling, both coefficients sharply decrease below $50 \mathrm{~K}$ and finally change sign from positive to negative at low temperature ${ }^{28}$. This supports the scenario of a Fermi-surface reconstruction that leads to a small, closed and coherent electron pocket, and it points to the same underlying mechanism related to the $\mathrm{CuO}_{2}$ plane, because this is the only common structural unit of the two compounds (Fig. 1). In Y123, the Fermi-surface reconstruction is consistent with bi-axial CDW order that competes with superconductivity ${ }^{14,23-25}$. Our data for Hg1201 do not allow us to extract the number of pockets, although it seems likely that there exists one pocket per $\mathrm{CuO}_{2}$ plane.

A recent analysis that spanned a broad doping and temperature range for a number of cuprates, including Y123 and Hg1201, determined the universal zero-field sheet resistance associated with the nodal states ${ }^{1}$. Below the pseudogap temperature $T^{*}$, where neutron diffraction measurements show the onset of an unusual magnetic state in both Y123 and Hg1201 (ref. 29), it was demonstrated that there exists another characteristic temperature $\left(T^{* *}\right)$ below which the planar transport becomes Fermi-liquid like ${ }^{1,2}$. For Y123, the temperature below which the CDW correlations are found approximately coincides with $T^{* *}$. Although CDW correlations remain to be reported for Hg1201, the similarity of the transport properties of the two compounds is remarkable. A central question is the relationship between the Fermi-surface reconstruction suggested by quantum oscillation measurements, the CDW correlations observed already in the zero-field high-temperature Fermi-liquid-like state, and the opening of the pseudogap at $T^{*}$ (refs 8,9 ). Our results rule out theories that relate the reconstruction of the Fermi surface to a unidirectional character of the crystallographic structure, and they imply that the applicability of theoretical models based on the $\mathrm{CuO}_{2}$ planes can now be thoroughly tested in a structurally simple compound, for example, through an extension of the present measurements to different doping levels. Our 


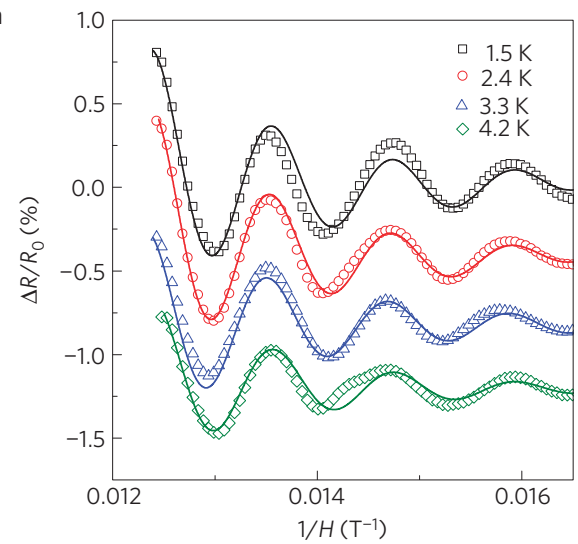

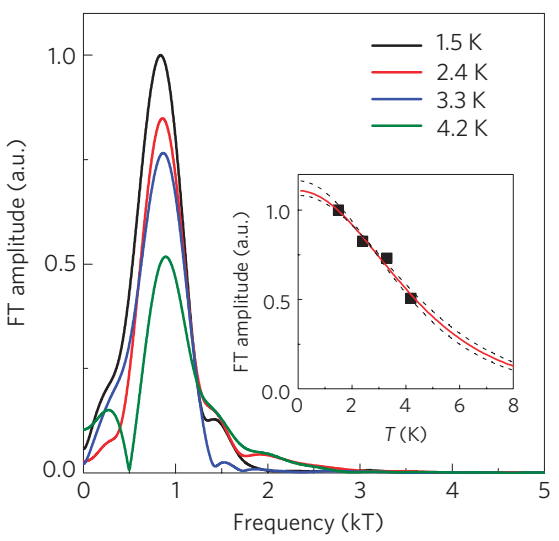

Figure 3 | Frequency of oscillations and effective mass. a, Oscillatory part of the magnetoresistance (obtained by subtracting a monotonic contribution) versus inverse field (symbols). Solid lines correspond to the Lifshitz-Kosevich fit (see Supplementary Information). b, Fourier transform (FT) of the oscillatory part, from which only one peak is observed, at $F=840 \pm 30 \mathrm{~T}$, with a temperature-independent position. Inset: in accordance with the Lifshitz-Kosevich formula, the temperature dependence of the amplitude yields a cyclotron mass of $m^{*}=(2.45 \pm 0.15) m_{\mathrm{e}}$. Solid red line: fit. Black dashed lines: error range. See Supplementary Information for details of the analysis.

Table 1 | Evolution of the Fermi surface.

\begin{tabular}{lllllll} 
& $\boldsymbol{T}_{\mathrm{c}}(\mathrm{K})$ & $\boldsymbol{p}$ & $\boldsymbol{F}(\mathrm{T})$ & $\boldsymbol{p}_{\text {eff }}$ & $\boldsymbol{m}^{*}\left(\boldsymbol{m}_{\mathrm{e}}\right)$ & Hall sign \\
\hline Hg1201 & 72 & 0.09 & $840 \pm 30$ & $0.061 \pm 0.002$ & $2.45 \pm 0.15$ & $(-;$ ref. 28) \\
Y123 (ref. 12) & 57.5 & 0.10 & $530 \pm 20$ & $0.038 \pm 0.002$ & $1.9 \pm 0.1$ & $(-;$ ref. 28) \\
Y124 (ref. 13) & 80 & 0.125 & $660 \pm 15$ & $0.047 \pm 0.002$ & $3.0 \pm 0.3$ & $(-;$ ref. 13) \\
T12201 (ref. 21) & 15 & 0.30 & $18,100 \pm 50$ & $1+p=1.3$ & $5 \pm 1$ & $(+;$ ref. 15) \\
\hline
\end{tabular}

Quantum oscillations have been observed in underdoped $\mathrm{Hg} 1201, \mathrm{Y} 123$ and $\mathrm{Y} 124$, as well as in overdoped TI2201. The first two columns list the respective superconducting transition temperatures ( $T_{\mathrm{C}}$ ) and doped hole concentrations $p$ (per planar copper atom). Coefficients extracted from quantum oscillation experiments are: $F-$ oscillation frequency; $p_{\text {eff }}-$ carrier density per pocket; $m^{*}-$ cyclotron mass, where $m_{\mathrm{e}}$ is the free-electron mass. The last column gives the sign of the Hall coefficient measured in high fields and low temperatures. In underdoped compounds $p_{\text {eff }}$ is substantially smaller than that expected from band-structure calculations $(1+p)$. See Supplementary Information for further information for $\mathrm{Hg} 1201$.

work furthermore demonstrates that the comparative quantitative study of $\mathrm{Hg} 1201$ and Y123 allows the determination of essential universal properties, and thus serves as a basis for a comprehensive understanding of the cuprates.

\section{Received 5 August 2013; accepted 30 September 2013; published online 10 November 2013}

\section{References}

1. Barišić, N. et al. Universal sheet resistance and revised phase diagram of the cuprate high-temperature superconductors. Proc. Natl Acad. Sci. USA 110, 12235-12240 (2013)

2. Mirzaei, S. I. et al. Spectroscopic evidence for Fermi liquid-like energy and temperature dependence of the relaxation rate in the pseudogap phase of the cuprates. Proc. Natl Acad. Sci. USA 110, 5774-5778 (2013).

3. Gor'kov, L. P. Kinetics of excitations on the Fermi arcs in underdoped cuprates at low temperatures. Phys. Rev. B 88, 041104(R) (2013).

4. LeBoeuf, D. et al. Thermodynamic phase diagram of static charge order in underdoped $\mathrm{YBa}_{2} \mathrm{Cu}_{3} \mathrm{O}_{\gamma}$. Nature Phys. 9, 79-83 (2013).

5. Riggs, S. C. et al. Heat capacity through the magnetic-field-induced resistive transition in an underdoped high-temperature superconductor. Nature Phys. 7, 332-335 (2011).

6. Senthil, T. \& Lee, P. A. Synthesis of the phenomenology of the underdoped cuprates. Phys. Rev. B 79, 245116 (2009).

7. Banerjee, S., Zhang, S. \& Randeria, M. Theory of quantum oscillations in the vortex-liquid state of high- $T_{\mathrm{c}}$ superconductors. Nature Commun. 4, 1700 (2013).

8. Efetov, K. B., Meier, H. \& Pépin, C. Pseudogap state near a quantum critical point. Nature Phys. 9, 442-446 (2013).

9. Sachdev, S. \& La Placa, R. Bond order in two-dimensional metals with antiferromagnetic exchange interactions. Phys. Rev. Lett. 111, 027202 (2013).

10. Yao, H., Lee, D. H. \& Kivelson, S. Fermi-surface reconstruction in a smectic phase of a high-temperature superconductor. Phys. Rev. B 84, 012507 (2011).
11. Chakravarty, S. \& Kee, H. Y. Fermi pockets and quantum oscillations of the Hall coefficient in high-temperature superconductors. Proc. Natl Acad. Sci. USA 105, 8835-8839 (2008).

12. Doiron-Leyraud, N. et al. Quantum oscillations and the Fermi surface in an underdoped high- $T_{\mathrm{c}}$ superconductor. Nature 447, 565-568 (2007).

13. Yelland, E. A. et al. Quantum oscillations in the underdoped cuprate $\mathrm{YBa}_{2} \mathrm{Cu}_{4} \mathrm{O}_{8}$. Phys. Rev. Lett. 100, 047003 (2008).

14. Sebastian, S. E., Harrison, N. \& Lonzarich, G. G. Towards resolution of the Fermi surface in underdoped high- $T_{\mathrm{c}}$ superconductors. Rep. Prog. Phys. 75, 102501 (2012).

15. Vignolle, B., Vignolles, D., Julien, M-H. \& Proust, C. From quantum oscillations to charge order in high- $T_{\mathrm{c}}$ copper oxides in high magnetic fields. C. R. Phys. 14, 39-52 (2013).

16. Carrington, A. \& Yelland, E. A. Band-structure calculations of Fermi-surface pockets in ortho-II $\mathrm{YBa}_{2} \mathrm{Cu}_{3} \mathrm{O}_{6.5}$. Phys. Rev. B 76, 140508(R) (2007).

17. Das, T. Electron-like Fermi surface and in-plane anisotropy due to chain states in $\mathrm{YBa}_{2} \mathrm{Cu}_{3} \mathrm{O}_{7-\delta}$ superconductors. Phys. Rev. B 86, 064527 (2012).

18. Elfimov, I. S., Sawatzky, G. A. \& Damascelli, A. Theory of Fermi-surface pockets and correlation effects in underdoped $\mathrm{YBa}_{2} \mathrm{Cu}_{3} \mathrm{O}_{6.5}$. Phys. Rev. B 77, 060504(R) (2008)

19. Nakamae, S. et al. Electronic ground state of heavily overdoped nonsuperconducting $\mathrm{La}_{2-x} \mathrm{Sr}_{x} \mathrm{CuO}_{4}$. Phys. Rev. B 68, 100502 (2003).

20. Platé, M. et al. Fermi surface and quasiparticle excitations of overdoped $\mathrm{Tl}_{2} \mathrm{Ba}_{2} \mathrm{CuO}_{6+\delta}$. Phys. Rev. Lett. 95, 077001 (2005)

21. Vignolle, B. et al. Quantum oscillations in an overdoped high- $T_{\mathrm{c}}$ superconductor. Nature 455, 952-955 (2008).

22. Kwak, J. F. et al. Magnetic quantum oscillations in tetramethyltetraselenafulvalenium hexafluorophosphate [(TMTSF) ${ }_{2} \mathrm{PF}_{6}$. Phys. Rev. Lett. 46, 1296-1299 (1981).

23. Wu, T. et al. Magnetic-field-induced charge-stripe order in the high-temperature superconductor $\mathrm{YBa}_{2} \mathrm{Cu}_{3} \mathrm{O}_{y}$. Nature 477, 191-194 (2011)

24. Ghiringhelli, G. et al. Long-range incommensurate charge fluctuations in (Y,Nd) $\mathrm{Ba}_{2} \mathrm{Cu}_{3} \mathrm{O}_{6+x}$. Science 337, 821-825 (2012). 
25. Chang, J. et al. Direct observation of competition between superconductivity and charge density wave order in $\mathrm{YBa}_{2} \mathrm{Cu}_{3} \mathrm{O}_{6.67}$. Nature Phys 8, 871-876 (2012).

26. Zhao, X. et al. Crystal growth and characterization of the model high-temperature superconductor $\mathrm{HgBa}_{2} \mathrm{CuO}_{4+\delta}$. Adv. Mater. 18, 3243-3247 (2006).

27. Barišić, N. et al. Demonstrating the model nature of the high-temperature superconductor $\mathrm{HgBa}_{2} \mathrm{CuO}_{4+\delta}$. Phys. Rev. B 78, 054518 (2008).

28. Doiron-Leyraud, N. et al. Hall, Seebeck, and Nernst coefficients of underdoped $\mathrm{HgBa}_{2} \mathrm{CuO}_{4+\delta}$ : Fermi-surface reconstruction in an archetypal cuprate superconductor. Phys. Rev. X 3, 021019 (2013).

29. Li, Y. et al. Unusual magnetic order in the pseudogap region of the superconductor $\mathrm{HgBa}_{2} \mathrm{CuO}_{4+\delta}$. Nature 455, 372-375 (2008).

30. Eisaki, H. et al. Effect of chemical inhomogeneity in bismuth-based copper oxide superconductors. Phys. Rev. B 69, 064512 (2004).

\section{Acknowledgements}

We thank S. Barišić, L. P. Gor'kov, M.-H. Julien, D. van der Marel, S. Sachdev and L. Taillefer for useful discussions. N.B. acknowledges F. Rullier-Albenque for sharing her insights into the transport of the cuprates. The work at the University of Minnesota was supported by the Department of Energy, Office of Basic Energy Sciences. The work in Toulouse was supported by the French ANR SUPERFIELD, Euromagnet II, and the LABEX NEXT. The work in Zagreb was supported by the Unity through Knowledge Fund. N.B. acknowledges support though a Marie Curie Fellowship.

\section{Author contributions}

N.B., C.P. and M.G. conceived the experiment. N.B., S.B., M.K.C., B.V., J.B. and C.P. performed the high-field transport measurements. N.B., M.K.C., C.D., W.T., G.Y. and X.Z. prepared the samples (crystal growth, annealing, magnetization and transport characterization, contacts). S.B., B.V. and C.P. analysed the data. N.B., C.P. and M.G. wrote the manuscript with input from all authors.

\section{Additional information}

Supplementary information is available in the online version of the paper. Reprints and permissions information is available online at www.nature.com/reprints.

Correspondence and requests for materials should be addressed to N.B., C.P. or M.G.

\section{Competing financial interests}

The authors declare no competing financial interests. 